\title{
FutureJournal
}

\section{Análise do Constructo Decisão Estratégica a partir de um Esquema de Classificação Multidimensional do Fenômeno}

Eduardo Guedes Villar

Professor do Programa de Pós-Graduação Lato Sensu da Universidade do Oeste de Santa Catarina, UNOESC, Brasil

eduardogvillar@gmail.com

\section{Queila Regina Souza Matitz}

Professora da Universidade Federal do Paraná, UFPR, Brasil queila.matitz@gmail.com

Karina De Déa Roglio

Professora da Universidade Federal do Paraná, UFPR, Brasil karinaroglio@gmail.com

\section{RESUMO}

Neste trabalho, o objetivo consiste em ampliar a compreensão das potencialidades e das limitações relacionadas ao uso do constructo decisão estratégica, a partir de uma análise multidimensional do fenômeno. Metodologicamente, além do levantamento bibliográfico utilizado para a construção do referencial teórico, foi desenvolvido um esquema de classificação multidimensional desse constructo decisão estratégica por meio da análise categorial temática de conteúdo realizada em um corpus textual formado por artigos selecionados a partir da literatura da área. Finalmente, a partir da descrição e análise das dimensões propostas - estrutura da decisão, importância da decisão, dificuldade da decisão, interação com outras decisões, participação na decisão e efetividade da decisão -, foram discutidas aplicações do esquema de classificação multidimensional do constructo decisão estratégica sob o ponto de vista (i) das formas de conceituação e operacionalização do fenômeno e (ii) da construção de tipologias de decisão estratégica. Incluem-se, entre as principais contribuições do esquema proposto, seu potencial em termos de: (i) esclarecimento de limitações relacionadas às formas tradicionais de conceituação e de operacionalização do constructo decisão estratégica em pesquisas da área; (ii) desenvolvimento de novos conceitos de decisão estratégica de natureza 


\section{FutureJournal}

multidimensional; (iii) integração entre as perspectivas de conteúdo e de processo estratégico; (iv) desenvolvimento de novas possibilidades de desenhos de pesquisa com foco na decisão estratégica e em processo decisório estratégico; desenvolvimento de novas tipologias de decisão estratégica.

PALAVRAS-CHAVE: Decisão. Estratégia. Processo decisório. Análise multidimensional.

\section{Analysis of the Construct Strategic Decision from a Classification Scheme of Multidimensional Phenomenon}

\section{ABSTRACT}

The objective of this study consists of broadening the understanding of the potentialities and limitations related to the use of the construct strategic decision, from a multidimensional analysis of the phenomenon. Methodologically, in addition to the literature survey used for the construction of the theoretical framework, we developed a classification scheme of the multidimensional construct strategic decision by means of categorical thematic analysis of content in a textual corpus consisting of articles selected from the literature of the area. Finally, from the description and analysis of the dimensions proposed - structure of the decision, importance of the decision, difficulty of the decision, interaction with other decisions, participation in decision-making and effectiveness of decision - it was discussed applications of multidimensional classification scheme of the construct strategic decision from the point of view (i) the forms of conceptualization and operationalization of the phenomenon and (ii) the construction of strategic decision making typologies. It is included, among the main 


\section{FutureJournal}

FUTURE STUDIES RESEARCH JOURNAL:

TRENDS AND STRATEGIES

contributions of the proposed scheme, its potential in terms of: (1) clarification of limitations related to traditional forms of conceptualization and operationalization of the construct strategic decision in researches of the area; (ii) development of new concepts of strategic decision of multidimensional nature; (iii) integration between the content prospects and strategic process; (iv) development of new possibilities of research designs with focus on strategic decision making and in the decision-making process; ( $v$ ) the development of new types of strategic decision making.

KEY-WORDS: decision, strategy, decision process, dimensional analysis. 


\section{INTRODUÇÃO}

Os temas decisão estratégica e processo decisório estratégico destacam-se nos estudos do campo de estratégia em face de sua importância na definição dos rumos, objetivos e resultados organizacionais (Eisenhardt, 1999; Nutt, 2011). Pesquisas em processo decisório no contexto organizacional são encontradas a partir da década de 1950, período no qual se introduziu o tema no contexto da abordagem do planejamento estratégico. Desde então, produziram-se pesquisas, teorias e abordagens diversas sobre o assunto, as quais resultaram em um campo teoricamente disperso em torno de uma ampla quantidade de entendimentos a respeito dos modos de conceituação e operacionalização da decisão no contexto gerencial (Langley, Mintzberg, Pitcher, Posada \& Saint-Macary, 1995; Nutt \& Wilson, 2010; Nutt, 2011). Entretanto, apesar do protagonismo e do expressivo avanço científico em torno da temática, esses estudos são embasados, frequentemente, em suposições incompletas a respeito do fenômeno (Eisenhardt \& Zbaracki, 1992).

Historicamente, dentre os fatores limitantes identificados em pesquisas da área, destaca-se a reificação da decisão. Ao apontarem para esse problema, Langley et al. (1995) destacaram dificuldades ontológicas e metodológicas relacionadas aos estudos sobre decisão estratégica na medida em que a decisão "não existe" de forma objetiva e concreta na realidade. Em decorrência dessa característica, os estudos em torno do tema demonstram dificuldades conceituais decorrentes, por exemplo, da confusão entre duas possibilidades de status ontológico do fenômeno: decisão como atribuição retrospectiva feita por um observador a partir da constatação de determinados efeitos provocados pela decisão e decisão como realidade empírica caracterizada pelo momento de escolha que antecede a ação (Mintzberg \& Waters, 1990; Einsenhardt \& Zbaracki, 1992; Tsoukas, 2010).

Outro problema de natureza metodológica é apontado por Papadakis, Thanos e Barwise (2010). Os autores verificaram diversidade nas definições e nas formas de operacionalização do conceito decisão estratégica em 46 
estudos empíricos de processo decisório publicados entre 1998 e 2008 em top journals dos EUA e Europa e apontam para a necessidade de ampliar o cuidado na conceituação, operacionalização e mensuração do constructo em pesquisas científicas. Quanto às formas de conceituação, foram identificados termos alternativos para descrever constructos relacionados à decisão, o que gera conflitos na literatura de processo decisório. Quanto às formas de operacionalização, foi constatada a necessidade de (i) utilização de amostras mais robustas capazes de suportar testes estatísticos avançados (por exemplo, análise fatorial confirmatória e modelagem de equações estruturais) e (ii) uso de desenhos de pesquisa longitudinais, adequados à exploração de relações entre contexto, processo de decisão, implementação, conteúdo e resultado (Papadakis et al., 2010).

Budde, Nagler e Friedli (2015), complementarmente aos problemas apontados acima, indicam uma tendência de supersimplificação em parcela expressiva das abordagens de processo decisório voltadas à construção e à aplicação de teorias na prática gerencial. Os autores salientam que as pesquisas na área, via de regra, não levam em consideração os múltiplos elementos de nível individual e organizacional envolvidos na tomada de decisão e suas interdependências, além de aspectos como complexidade e dinamismo do ambiente. Shepherd e Rudd (2014) comprovam esse argumento ao destacarem características tais como inconsistência de resultados e entendimento fragmentado nas pesquisas sobre decisão estratégica, ambas decorrentes da falta de uma visão mais abrangente em relação aos múltiplos fatores envolvidos no contexto da decisão.

Entretanto, independentemente de dificuldades e desafios na tentativa de definir e descrever o fenômeno, Franz e Kramer (2010) defendem o aprofundamento dos estudos de decisão estratégica, conforme apontado anteriormente por Dean e Sharfman (1996). Nutt (2011), por exemplo, sugere que a prática usual de denominar uma decisão de "estratégica", como forma de conferir-Ihe importância e significado, é indevida e sugere a realização de estudos futuros com o propósito de ampliar o poder de explicação de decisões efetivamente relacionadas à ação estratégica. Ainda segundo o autor, diversos esforços de pesquisa têm adicionado peças ao quebra-cabeças denominado campo de estudos sobre 
decisão estratégica, entretanto são poucas as tentativas de procurar as peças faltantes.

O objetivo geral deste trabalho, considerando-se os argumentos expostos anteriormente, consiste em ampliar a compreensão das potencialidades e das limitações relacionadas ao uso do constructo decisão estratégica, a partir de uma análise multidimensional do fenômeno. Como objetivos específicos, incluem-se: (i) descrever aplicações e limitações conceituais e operacionais relacionadas ao uso do constructo decisão estratégica apontadas na literatura da área; (ii) identificar dimensões constitutivas do constructo decisão estratégica a partir de análise da literatura da área; (iii) desenvolver um esquema de classificação multidimensional do constructo decisão estratégica; e (iv) discutir aplicações do esquema de classificação multidimensional do constructo decisão estratégica.

Justifica-se o estudo aqui proposto por representar um esforço teórico incremental potencialmente útil na acumulação de conhecimentos a respeito do tema proposto, especialmente no esclarecimento de aspectos a serem considerados na conceituação e na operacionalização do constructo decisão estratégica. Conforme os trabalhos mencionados anteriormente, há necessidade de desenvolvimento de propostas teóricas mais complexas relacionadas ao significado do fenômeno. Esforços dessa natureza podem contribuir, por exemplo, para ampliar a compreensão a respeito de um tema central no campo de estudos de estratégia e para o desenvolvimento de teorias mais robustas e potencialmente úteis à construção de práticas administrativas coerentes com as necessidades organizacionais.

Estruturou-se este ensaio teórico em cinco seções. Na introdução, foram apresentados as justificativas e os objetivos do trabalho. Na segunda seção, foram descritas aplicações e limitações conceituais e operacionais relacionadas ao uso do constructo decisão estratégica conforme apontadas na literatura da área. Na terceira seção, foram apresentados os procedimentos metodológicos e os resultados da análise multidimensional do constructo decisão estratégica. Na quarta seção, foram discutidas aplicações do esquema de classificação multidimensional do constructo decisão estratégica sob o ponto de vista das formas de conceituação e 
operacionalização do fenômeno e da construção de tipologias de decisão estratégica. Na quinta e última seção, foram apresentadas considerações finais, nas quais se destacam as contribuições da pesquisa e indicações de trabalhos futuros.

\section{O CONSTRUCTO DECISÃO ESTRATÉGICA NA LITERATURA DA ÁREA: APLICAÇõES E LIMITAÇõES}

Nesta seção, descrevem-se algumas formas de conceituação e de operacionalização do constructo decisão em trabalhos anteriores das áreas de estratégia e de estudos organizacionais. Embora não se trate de uma revisão exaustiva, o conteúdo contribui para ampliar a compreensão atual do fenômeno decisão e aponta para limitações das pesquisas da área.

Decisão é um conceito amplamente aceito no campo das teorias organizacionais (Mintzberg \& Waters, 1990). Contudo, Barnard (1968) já apontava para a dificuldade de localizar ou revelar as decisões na medida em que estas "não existem". Há, portanto, desde a origem dos estudos de administração estratégica, um reconhecimento da natureza intangível das decisões, as quais não se manifestam de maneira independente e concreta na realidade. Aliás, parte dos problemas encontrados ao estudar decisões estratégicas é decorrente da própria natureza intangível do fenômeno (Pettigrew, 1990).

Entretanto, a dificuldade de apreensão do fenômeno decisão não impediu o reconhecimento de sua importância e de algumas das formas pelas quais se manifesta. Yates (1990), por exemplo, verificou algum grau de consenso quanto à existência de diferentes tipos de decisões. As decisões denominadas estratégicas, segundo Pennings (1985), seriam significativas, não estruturadas, complexas, coletivas e consecutivas. Seriam, ainda, formais ou informais, pretendidas ou emergentes. Hickson, Butler, Cray, Mallory e Wilson (1986) sugerem diferentes tipos de processo de decisão, dentre os quais os processos (i) esporádicos (não contínuos), (ii) fluidos (contínuos e suaves) e (iii) constritos (restritos a uma pequena parcela de grupos de interesse e altamente político). Papadakis e Barwise (1998) também apresentam uma definição clássica de decisão estratégica. 
De acordo com os autores, as decisões estratégicas em geral são caracterizadas (i) por alto grau de abrangência, risco, dificuldade de reversão e efeitos de longo prazo, (ii) por criarem uma ponte entre as estratégias deliberadas e emergentes, (iii) por se tornarem uma das principais fontes de aprendizagem organizacional, (iv) por terem papel central no desenvolvimento individual dos gestores e (v) por sua transversalidade em relação a diferentes funções e disciplinas acadêmicas. Para Elbanna (2006), decisões estratégicas, no âmbito organizacional, seriam caracterizadas pela origem em indivíduos localizados no topo hierárquico da organização. Consequentemente, estariam sujeitas à influência de um conjunto de fatores psicológicos, culturais e políticos relacionados a esses indivíduos. A esse respeito, Pettigrew (1992) já havia considerado a pressão de fatores ambientais externos como importante elemento condicionante das decisões de altos executivos.

Ainda a respeito das decisões estratégicas, Wilson (2003) aponta uma dificuldade em relacionar desempenho e decisões na medida em que uma decisão, em geral, está relacionada simultaneamente a múltiplos problemas ou oportunidades e riscos. Há, portanto, probabilidade de associação entre uma decisão e múltiplos fatores organizacionais. As consequências de uma decisão, considerando-se tal contexto, nem sempre podem ser isoladas e compreendidas de forma linear. Adicionalmente, decisões podem estar relacionadas a outras decisões, as quais podem ser antecedentes, subsequentes, concomitantes e concorrentes (Langley et al., 1995).

Quanto às diferentes formas de operacionalização do constructo decisão estratégica, é útil lembrar que estudos de estratégia têm sido divididos tradicionalmente em duas categorias: conteúdo e processo. Pesquisas sobre conteúdo estratégico têm como foco a própria decisão e abordam questões relacionadas a diferentes tipos de decisão estratégica e suas consequências, dentre essas: diversificações, aquisições e fusões, internacionalização, estratégias competitivas, etc. Pesquisas sobre processo estratégico, por outro lado, buscam explicar a forma como são formuladas e implementadas as decisões estratégicas. A forma de operacionalização do constructo, portanto, depende do interesse da pesquisa: em conteúdo, em 
processo estratégico ou em ambos. Enquanto pesquisas com foco em conteúdo estratégico buscam operacionalizar a decisão de acordo com o significado de estratégia associado ao termo, pesquisas com foco em processo estratégico buscam acompanhar o desenvolvimento do processo decisório.

Em revisão da própria produção, Mintzberg e Waters (1990) perceberam que suas pesquisas não tratavam definitivamente de fluxos de decisões, mas de ações. Teoricamente, a observação das ações permitiria encontrar os vestígios de decisão (choice) deixados ao longo do tempo pelas organizações. Entretanto, conforme os próprios autores apontaram, o pressuposto de que a decisão precede a ação nem sempre é verdadeiro. $\mathrm{E}$ concluem que a comunidade acadêmica, ao tentar operacionalizar o conceito de decisão, frequentemente incorre nesse equívoco, por exemplo, ao restringir os estudos à lógica da "máquina da burocrática", esquecendose do fenômeno em si (Mintzberg \& Waters, 1990). Chia (1994) critica o trabalho de Mintzberg e Waters (1990) por tratar-se, em sua opinião, de uma discussão superficial a respeito do significado da palavra "escolha" (choice do original), em detrimento da busca por razões mais profundas relacionadas à dificuldade de pesquisar o fenômeno decisão.

O trabalho de Pettigrew (1990) ilustra a noção de decisão estratégica alinhada a uma integração entre as ideias de conteúdo e de processo estratégico. Para o autor, a análise da mudança (change) poderia ser uma alternativa para ampliar o entendimento do fenômeno estratégia no contexto organizacional, até então restrito às decisões (choices) como episódios isolados. Em suas palavras, "normalmente [a decisão é considerada como um fenômeno] não-histórico, não-processual e nãocontextual" (Pettigrew, 1990, p. 9). Tratava-se a decisão, portanto, como uma unidade de análise isolada, um evento único, o qual é, frequentemente, separado de outros eventos imediatos que o circundam e também de outros mais longínquos capazes de conferir-lhe forma, significado e conteúdo. Para o autor, a análise da(s) mudança(s) organizacional(is) permitiria captar o fenômeno por meio da análise de múltiplos níveis de análise e de suas interconexões ao longo do tempo. Sua proposta consistia na inclusão de preocupações - por parte dos 
pesquisadores - relacionadas ao contexto no qual as decisões (changes) ocorrem, aos múltiplos níveis de análise envolvidos e à conectividade entre esses. Seria necessária, portanto, uma integração temporal entre passado, presente e futuro, por meio da qual se construiria a trajetória organizacional. Haveria, ainda, múltiplas formas de investigação disponíveis para estudar decisões estratégicas, uma vez que essas poderiam ser observadas como processo, estrutura ou resultado ou, ainda, discutidas com ênfase prescritiva ou descritiva.

As considerações apresentadas demonstram algumas das dificuldades envolvidas na operacionalização do constructo decisão abordadas por Nutt (2011) e Nutt e Wilson (2010). Nutt (2011) ressalta a necessidade de atenção para a unidade de análise. Para o autor, estudos de decisão implicam a consideração de uma gama completa de ações que podem ocorrer durante o processo de decisão tais como, por exemplo, reconhecimento do problema, diagnóstico, busca por alternativas, avaliação. Contudo, frequentemente as pesquisas utilizam apenas a escolha (choice) como unidade de análise, reduzindo a decisão a uma comparação entre alternativas. Nutt (2011) também critica os estudos de conteúdo estratégico pela forma genérica como o termo decisão estratégica tem sido usado e defende a importância de explicitar-se o tipo de decisão sob estudo em trabalhos futuros. Quanto às pesquisas com foco em processo decisório, Nutt e Wilson (2010) apontam que, a partir da década de 1980, cresce o interesse dos pesquisadores no mapeamento do progresso das decisões estratégicas e, em adição, no desenvolvimento de explicações a respeito dos processos estratégicos. Destacam-se as pesquisas realizadas por Hickson et al. (1986), nas quais se revela um interesse contínuo em descrever as características do processo de decisão, assim como em identificar associações entre decisões e resultado organizacional.

Apesar da classificação tradicional das pesquisas em termos de conteúdo e processo estratégico, Butler (1990) já apontava para a importância da relação entre o tipo de conteúdo da decisão e o processo de tomada de decisão. Portanto, apesar de alguns estudos clássicos na área terem comparado o processo de decisão desconsiderando o conteúdo das decisões sob análise - por exemplo, as 25 decisões do estudo de Mintzberg, 
Raisinghani e Théorêt (1988), as 150 decisões de Hickson et al. (1986) e as 376 decisões de Nutt $(1998 ; 2001)$-, deve-se observar como o conteúdo da decisão impacta o processo de tomada da decisão, e vice e versa (Mintzberg \& Waters, 1990), na medida em que as duas categorias são complementares e não opostas ou concorrentes (Elbanna, 2006).

Quanto ao nível de análise utilizado nas pesquisas de decisão estratégica, enquanto alguns autores têm considerado a decisão em nível organizacional (Dean \& Sharfman, 1996; Mintzberg, Otis, Shamsie \& Waters, 1988), outros estudam a decisão em nível individual (Hambrick, Finkelstein, \& Cannella Jr., 2009). Decisões no nível organizacional, como no estudo de Mintzberg et al. (1988), são caracterizadas pelo fato de a organização iniciar com um entendimento incipiente a respeito da situação de decisão, das alternativas de escolha e de possíveis processos de tomada de decisão. No nível individual, o estudo de Hambrick e Manson (1984) exemplifica pesquisas nas quais as decisões são tomadas a partir de uma orientação interna do decisor ou de um conjunto de decisores (top management team). Dentre os fatores individuais potencialmente influentes na decisão em nível individual, é possível destacar, por exemplo: (i) valores e cognição (Hambrick \& Mason, 1984) e (ii) fatores psicológicos e experiências observáveis (Hambrick et al., 2009).

As discussões apresentadas demonstram a diversidade nos aspectos potencialmente consideráveis na conceituação e operacionalização do fenômeno decisão estratégica, dentre esses: agente responsável pela decisão, grau de importância da decisão sob o ponto de vista individual e/ou organizacional, grau de estruturação da tomada de decisão, tipo e grau de participação de diferentes atores ou grupos organizacionais na tomada de decisão, grau de complexidade da decisão, grau de formalidade da tomada de decisão, tipo de resultado da decisão, tipo de processo de tomada de decisão, elementos antecedentes e/ou condicionantes do processo de tomada de decisão, interação da decisão com outras decisões, etc.

Com base nesse primeiro levantamento exploratório de potenciais dimensões do fenômeno, a próxima seção sistematiza e apresenta uma proposta de representação multidimensional do constructo decisão 
estratégica a partir de análise da literatura da área e conforme os objetivos propostos pelo trabalho.

\section{ANÁlise MULTIDIMENSIONAL DO CONSTRUCTO DECISÃO ESTRATÉGICA}

A análise exploratória inicial da literatura, conforme apresentada na seção 2, permitiu a identificação de potenciais dimensões a serem consideradas na conceituação e operacionalização do constructo decisão estratégica. Como forma de aprofundar e sistematizar a análise proposta, realizou-se uma análise categorial temática de conteúdo (Bardin, 2011), a partir de um corpus textual formado por artigos selecionados na base de dados Web of Knowledge. Os artigos foram identificados por meio de uma busca agregada pelos termos decisão estratégica (strategic decision, conceito (concept), dimensão (dimension), classificação (classification), tipologia (typology) e perspectiva (perspective) em resumos ou títulos de trabalhos publicados a partir de 1990. Também foram aplicados os filtros Social Sciences e Business and Economics. Como resultado, identificaram-se os artigos de Dean, Sharfman e Ford (1991), Chia (1994), Dean e Sharfman (1996), Harrison (1996), Harrison e Pelletier (1998), Dholakia e Bagozzi (2002), Elbanna (2006) e Franz e Kramer (2010). Outros artigos identificados por meio da busca foram excluídos com base nos seguintes critérios: a) tratavam de contextos/localidades geográficas específicas (ex. sudoeste da China, Emirados Árabes); b) consistiam em aplicações voltadas a áreas funcionais específicas (ex. cadeia de valor, comportamento do consumidor); ou c) focavam no uso de técnicas ou ferramentas de apoio à tomada de decisão (ex. árvore de decisão, decisão multicritério, decisão fuzzy).

Para auxiliar na delimitação das dimensões, optou-se por adotar alguns dos critérios propostos por Dean e Sharfman (1996): (i) as dimensões devem ser centrais na literatura de decisão estratégica, uma vez que a adoção de dimensões periféricas poderia levar a negligenciar a própria natureza do conceito; (ii) as dimensões devem ser lógica e teoricamente distintas. Foram considerados, ainda, os critérios para desenvolvimento de esquemas de classificação de base qualitativa conforme 
propostos por Bailey (1994): (i) as unidades ou entidades devem descrever aspectos fundamentais do fenômeno e devem ser agrupadas com base em suas similaridades; (ii) deve-se minimizar a variância intragrupo e maximizar a variância entre grupos; (iii) em conjunto, as categorias do esquema de classificação devem compor uma unidade sob o ponto de vista teórico, ou seja, devem apresentar relações entre si; (iv) as categorias do esquema de classificação devem exibir exaustividade (incluem classes apropriadas a todos os objetivos a serem classificados) e exclusividade (pressupõe-se a existência de apenas uma classe correta ou adequada para cada objeto); ( v) o esquema de classificação deve ser desenvolvido com foco na simplicidade de forma e de conteúdo, parcimônia ou redução da complexidade de fenômenos multidimensionais em níveis adequados ao desenvolvimento de descrições, análises e interpretações de dados; pertinência ou adequação do sistema de categorias às intenções da investigação; objetividade e fidelidade ou facilidade de replicação da classificação; produtividade do sistema de classificação em termos de inferências, dados e geração de novas propostas de pesquisas.

Na Figura 1, a seguir, ilustra-se o resultado da análise do constructo decisão estratégica, proposto a partir da análise da literatura, sob a forma de um esquema de classificação multidimensional. Em seguida, cada uma das dimensões está apresentada de forma descritiva. Como principal limitação metodológica, destaque-se que, apesar do esforço analítico, todo sistema de classificação de base qualitativa está sujeito a escolhas arbitrárias por parte de seu(s) autor(es). 


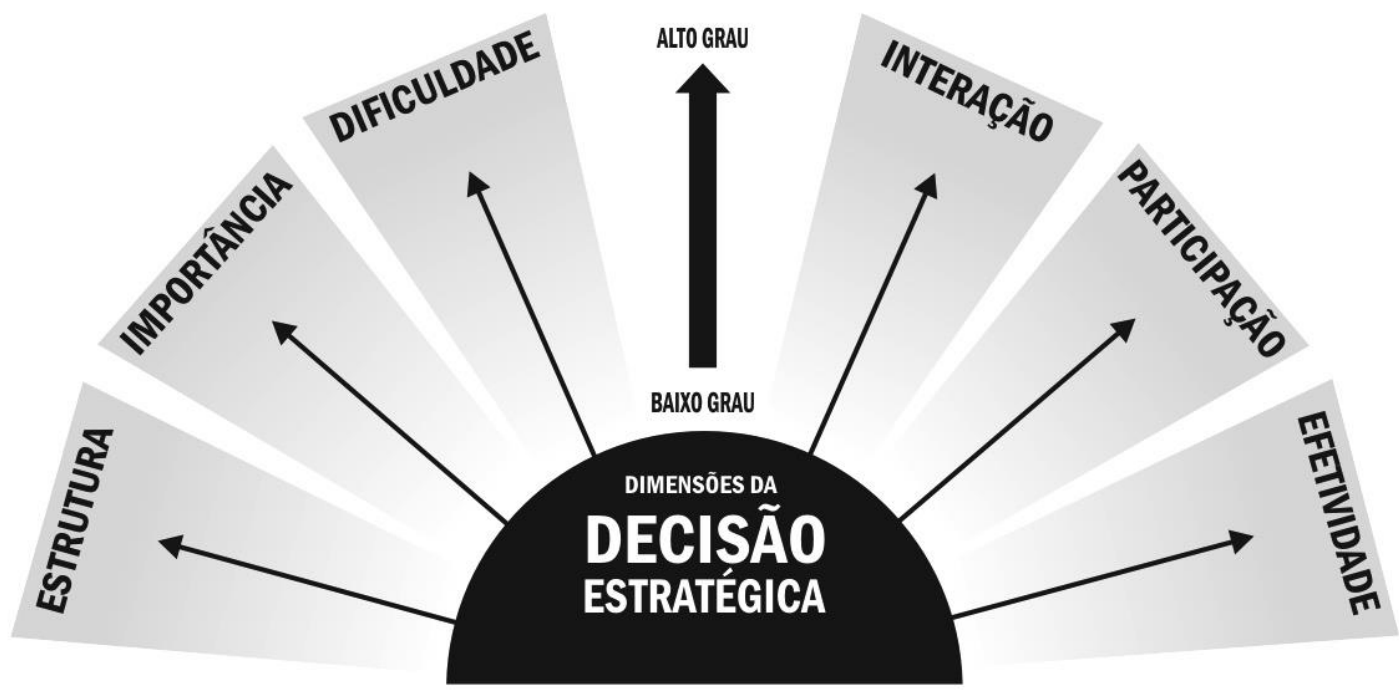

Figura 1: Esquema de classificação multidimensional do constructo decisão estratégica

Fonte: Elaborada pelos autores

\subsection{ESTRUTURA DA DECISÃO}

Quanto à estrutura da decisão - aqui entendida como grau de ordenação da tomada de decisão a partir de um conjunto distinguível e lógico de etapas dependentes entre si -, pode-se percorrer um continuum entre decisões altamente estruturadas e decisões não estruturadas ou amorfas. Tomadas de decisão altamente estruturadas seguem etapas predefinidas e fundamentadas na busca de racionalização do processo de tomada de decisão, enquanto tomadas de decisão amorfas são caracterizadas como uma confluência aleatória de eventos (Eisenhardt \& Zbaracki, 1992), sem apresentar estrutura ou sequência aparente (Langley et al., 1995).

Segundo Franz e Kramer (2010), a estrutura da decisão depende de sete elementos principais: (i) disponibilidade de informação; (ii) utilização de informação na tomada de decisão; (iii) confiança do decisor nos resultados; (iv) confiança na estabilidade da configuração do ambiente; (v) consistência do decisor; (vi) momento da decisão; e (vii) racionalidade empregada. 
A disponibilidade de informação afeta a estrutura da decisão na medida em que interfere na capacidade de compreensão do contexto, de formulação e de avaliação de alternativas. Embora March e Simon (1958) tenham questionado a visão do "homem econômico", capaz de acessar e interpretar todas as informações disponíveis e necessárias à one best decision, a teoria administrativa tem chamado a atenção para a importância das informações na tomada de decisão. Um alto grau de estruturação da decisão, portanto, depende da possibilidade de acesso à informação em termos de qualidade, quantidade e custos. Nutt e Wilson (2010) sugerem, ainda, a importância da confiabilidade e das fontes de informações como elementos a serem considerados na tomada de decisão gerencial.

O modo como as informações são consideradas pelo decisor também molda a estrutura da decisão. Moody (1983), por exemplo, define cinco elementos potencialmente considerados na decisão: fatos, conhecimento, experiência, análise e julgamento. Uma decisão na qual são consideradas essenciais informações tais como fatos, dados e conhecimentos, apresenta um grau de estruturação superior ao de uma decisão na qual esses elementos são considerados apenas marginalmente. E tanto a diversidade de elementos quanto a intensidade de uso das informações impactam a estrutura da decisão.

A confiança do decisor nos resultados diz respeito ao nível de segurança do indivíduo tomador de decisão em relação à qualidade da decisão em comparação a alternativas (Dholakia \& Bagozzi, 2002). Esse elemento interfere na estrutura da decisão na medida em que maior insegurança pode diminuir o grau de estruturação da decisão, e vice-versa.

Quanto à confiança do decisor na estabilidade da configuração do ambiente, Dean e Sharfman (1996) concluem pela existência de uma relação entre maior grau atribuído de estabilidade ao ambiente e maior estruturação da decisão. Por outro lado, em ambientes considerados instáveis, as decisões parecem tomar os próprios fluxos de ação (Dean \& Sharfman, 1996).

A consistência do decisor refere-se ao grau de esforço do indivíduo para moldar ou construir determinada decisão. Bandura (1995) denomina essa consistência de autoeficácia percebida, a qual consiste na crença do próprio decisor em seu empenho para organizar e executar os cursos de 
ação necessários para atingir os objetivos almejados. A consideração do esforço investido no processo pode, por si só, ser um sinal para o indivíduo a respeito do grau de significância do objetivo escolhido (Dholakia \& Bagozzi, 2002). Decisões mais estruturadas, em geral, estão relacionadas a maior grau de consistência ou autoeficácia do decisor.

$\mathrm{Na}$ análise do momento da decisão, Harrison e Pelletier (1998) indicam ser evidente que há um tempo ideal para tomar-se uma decisão estratégica. Segundo os autores, as decisões poderiam ser classificadas com base na possibilidade de escolha intencional - por parte do decisor do momento ideal de tomada de decisão, associado à maximização dos efeitos pretendidos. Assim, poderia qualificar-se um momento de decisão como bom ou ruim.

Quanto à racionalidade empregada, Simon (1987) afirma que a teoria em processo decisório estratégico necessita levar em conta tanto os processos racionais, quanto os intuitivos (ou não lógicos), pois, ao decidir, o gestor pode utilizá-los conjuntamente. No que se refere ao impacto da racionalidade empregada sobre a estrutura da decisão, verifica-se que decisões altamente racionais, ou seja, baseadas em dados e informações objetivas e construídas por meio lógico e analítico conferem maior grau de estruturação à decisão. Já decisões intuitivas, baseadas no gut-feeling do decisor, sugerem menor estruturação da decisão. Contudo, quanto à eficácia da decisão, verificou-se que a análise racional e as escolhas intuitivas são componentes complementares do processo decisório efetivo (Hodgkinson \& Clarke, 2007).

\subsection{IMPORTÂNCIA DA DECISÃO}

Quanto à importância da decisão - aqui entendida como valor atribuído à decisão no contexto organizacional em termos de abrangência ou impacto -, pode-se percorrer um continuum entre decisões altamente importantes e decisões não importantes. A uma decisão altamente importante é atribuído alto valor simbólico-social.

Moody (1983) sugere cinco fatores a serem considerados na análise da importância das decisões: (i) magnitude ou grau de compromisso assumido com a decisão, (ii) flexibilidade do planejamento da decisão, (iii) 
concretude dos objetivos e metas, (iv) capacidade de conexão das decisões com dados e informações quantificáveis, e (v) impacto humano da decisão. O primeiro fator indica uma relação direta entre grau de comprometimento de capital, quantidade de pessoas envolvidas e abrangência temporal dos impactos da decisão sobre o grau de importância da decisão. O segundo fator, flexibilidade do planejamento da decisão, diz respeito ao grau de reversibilidade do curso de ação tomado a partir da tomada de decisão. Decisões mais importantes seriam caracterizadas por baixo grau de reversibilidade. O terceiro fator indica que decisões tomadas em condições nas quais objetivos e metas estão devidamente estabelecidos têm menor impacto se comparadas a decisões tomadas em condições nas quais os objetivos são incertos ou flutuantes. O quarto fator indica uma relação entre grau de importância da decisão e embasamento em dados. Decisões mais importantes são tomadas com base em informações de difícil especificação e interpretação. O quinto e último fator relaciona o grau de importância da decisão à quantidade de pessoas sobre as quais exerce impacto.

Franz e Kramer (2010) identificaram a pressão da situação como um fator condicionante do grau de importância ou do valor atribuído a uma decisão no contexto organizacional. Segundo os autores, a pressão do tempo para decidir, seja uma pressão exercida pelo próprio decisor ou pelas pessoas envolvidas no processo, impacta na percepção da importância da decisão.

Outro aspecto relevante a ser considerado em relação à importância da decisão no contexto organizacional foi abordado por Dholakia e Bagozzi (2002). Os autores fazem uma distinção entre a importância da decisão e a importância do objetivo da decisão em termos de resultado para o próprio decisor. Enquanto a importância do objetivo refere-se ao valor subjetivo dos resultados conforme atribuído pelo decisor e de acordo com seus interesses particulares, a importância da decisão refere-se ao valor intrínseco da própria decisão, independentemente dos objetivos do decisor.

\subsection{DIFICULDADE DA DECISÃO}


Quanto à dificuldade da decisão - aqui entendida como grau de esforço envolvido na tomada de decisão ou no processo de tomada de decisão -, pode-se percorrer um continuum entre decisões de alto e baixo grau de dificuldade. A uma decisão difícil estão relacionados esforços de maior intensidade decorrentes de maior complexidade relacionada a procedimentos de coleta de informações, acesso a dados e informações relevantes, quantidade de informações e de pessoas envolvidas, fontes de incerteza, objetivos conflitantes, alternativas de solução, entre outros elementos.

Conforme Hickson et al. (1986, p.248), "para conhecer o processo de uma decisão, primeiro faz-se necessário avaliar a complexidade dos problemas e a 'politicidade' [do original politicality] dos interesses envolvidos". A análise da 'politicidade' será desenvolvida na subseção 3.5, relacionada à dimensão de participação na decisão. Portanto, nesta subseção, destaca-se a necessidade de estabelecer o grau de complexidade do problema ou situação à qual a decisão está relacionada, aqui denominada de dificuldade da decisão.

Clemen (1996) identificou quatro fontes de dificuldade na tomada de decisão: a) complexidade da situação da decisão: (a) diversidade e quantidade de questões/assuntos, fontes de dados, partes interessadas e/ou possibilidades de resultado/solução; b) incerteza com relação aos dados, eventos e/ou resultados futuros; c) existência de objetivos concorrentes; d) perspectivas variadas de solução. Além desses elementos, o número de alternativas a ser considerado é um fator condicionante da dificuldade da decisão. Harrison e Pelletier (1998) sugerem uma relação direta entre quantidade e qualidade de alternativas e o grau de dificuldade da decisão. Decisões são mais fáceis quando uma única alternativa está relacionada a consequências mais positivas em comparação a todas as demais alternativas possíveis. Além disso, os autores mencionam uma relação entre a quantidade de esforço despendido para geração de alternativas e a quantidade de alternativas a serem analisadas. Em geral, há alternativas a respeito das quais o decisor não tem consciência (Harrison \& Pelletier, 1998), assim como há alternativas que serão ignoradas sem a 
devida avaliação em decorrência de sua capacidade cognitiva limitada (Einsenhardt, 1989).

Dean e Sharfman (1996) mencionam a dificuldade de implementação da decisão como um indicador do grau de dificuldade da decisão. Enquanto informações relativas à execução são diferentes de uma decisão para outra, a efetividade de qualquer decisão deve levar em conta sua implementação. Complementarmente, Mintzberg e Waters (1990) apontam para os problemas relacionados à implementação das decisões na medida em que algumas intenções são traduzidas mais perfeitamente em ação do que outras. Assim, o grau de executabilidade torna-se um indicador da dificuldade da decisão.

\subsection{INTERAÇÃO COM OUTRAS DECISÕES}

Quanto às interações entre decisões - aqui entendidas como: (i) tipo de relações entre diferentes decisões em termos de hierarquia e ordenação do tempo, (ii) intensidade das relações entre diferentes decisões em termos de proximidade no tempo e grau de influência entre si e (iii) contexto de ocorrência das decisões em termos de proximidade, local, participantes envolvidos, recursos utilizados, etc. -, pode-se percorrer um continuum entre decisões com alto grau de interação e decisões com baixo grau de interação.

Mintzberg, Raisinghani e Théorêt (1976) indicaram que uma decisão estratégica cria ondas de decisões menores. Mais tarde, Langley et al. (1995) verificaram a existência de relações entre decisões na maioria dos contextos estudados e classificaram as ligações identificadas em: a) sequenciais, b) precursoras e/ou c) concorrentes. As ligações sequenciais consideram a inter-relação entre decisões relativas ao mesmo assunto em diferentes momentos, as quais podem se dar por aninhamento (quando a decisão maior envolve uma série de decisões menores), bola de neve (quando um conjunto de pequenas decisões leva à decisão de maior porte) e recorrente (quando a mesma decisão se repete em diferentes situações). As ligações precursoras dizem respeito a situações nas quais uma decisão afeta as premissas de decisões subsequentes relacionadas a diferentes 
assuntos. Neste tipo de ligação, uma decisão anterior pode permitir, evocar, impedir, desencadear e/ou mesclar decisões futuras. Finalmente, as ligações concorrentes consistem em situações nas quais se identificam decisões a respeito de diferentes assuntos agrupados (concorrem por recursos, participantes, tempo e energia dentro da organização) ou contextuais (estão imersas em organizações com as mesmas pessoas, estratégias e uma única cultura e, portanto, estão ligadas umas às outras).

Langley et al. (1995) verificam, ainda, cinco formas diferentes de acoplamento entre decisões, formando um continuum entre relações mais justas até relações mais dispersas. Em um dos extremos estão as decisões cujos assuntos são totalmente acoplados (do original fully-coupled) e correspondem àquelas nas quais uma questão parece determinar outros assuntos. Em geral, decisões com assuntos totalmente acoplados são encontradas em organizações caracterizadas por uma gestão centralizada em um único gestor ou empreendedor. Em organizações onde não há completa centralização de poder de decisão, podem existir decisões cujos assuntos são estilisticamente acoplados (do original stylistically-coupled). Nessa categoria são incluídas decisões realizadas a partir de um estilo ou perspectiva compartilhada pelos gestores envolvidos, com base em uma visão integrada da estratégia organizacional estabelecida. Decisões cujos assuntos são intricadamente acoplados em redes (do original intricatelycoupled) são comuns em organizações organizadas por projetos ou com densa interação horizontal entre diferentes unidades. Quando regras e procedimentos são os principais mecanismos de ligação entre assuntos de decisões, essas são denominadas de formalmente acopladas (do original formally-coupled). Este tipo de relação é observado em organizações com sistema de interação burocrático. Finalmente, decisões com assuntos fracamente acoplados (do original loosely-coupled) são caracterizadas pela baixa interação, embora concorram por recursos em comum.

\subsection{PARTICIPAÇÃO NA DECISÃO}

Quanto à participação na decisão - aqui entendida como quantidade e diversidade de interesses, percepções, papéis, graus de envolvimento ou opiniões consideradas na tomada de decisão -, pode-se percorrer um 
continuum entre decisões com alto grau de participação e decisões com baixo grau de participação. Decisões com alto grau de participação são caracterizadas por grande quantidade e diversidade de interesses, percepções ou opiniões envolvidas no processo de tomada de decisão.

De acordo com a literatura consultada, os estudos de decisão nos campos de estratégia e de estudos organizacionais têm focado prioritariamente a visão racional da decisão e conferido menor importância a outras características do fenômeno, tais como participação e política (Papadakis \& Barwise, 1998; Elbanna, 2006; Child, Elbanna, \& Rodrigues, 2010). Entretanto, há um componente político na tomada de decisão na medida em que as pessoas nas organizações apresentam diferentes interesses em razão de elementos funcionais, hierárquicos, profissionais e pessoais (Hickson et al., 1986). Além disso, no contexto organizacional, há evidências empíricas de tentativas de uso da influência pessoal sobre os resultados das decisões, de modo que interesses individuais sejam atendidos (Pfeffer, 1981; Dean \& Sharfman, 1996). Segundo Harrison e Pelletier (1998), por exemplo, a influência das preferências do gerente sobre as escolhas estratégicas (choices) é evidente em parte significativa dos casos observados. Child et al. (2010) indicam que a influência do poder e o status da organização podem ter impactos positivos e negativos sobre a tomada de decisão. Entre os impactos positivos incluem-se, por exemplo, consideração de diferentes pontos de vista, cuidado adicional no planejamento e definição das decisões e acompanhamento da adaptação organizacional às mudanças geradas pela tomada de decisão. Entre os impactos negativos, incluem-se, por exemplo: o compromisso não direto com a qualidade da decisão, menor coesão e comprometimento na implementação das decisões e atrasos e necessidade de esforços adicionais no processo decisório (Child et al., 2010).

Quanto ao papel das pessoas envolvidas na decisão, Franz e Kramer (2010) indicam duas funções: a do(s) indivíduo(s) que decide(m) e a dos colaboradores para a decisão. Ao decisor cabe a escolha final entre as diferentes alternativas possíveis, as decisões podem ser descritas como de âmbito individual, interpessoal, familiar, organizacional, de comunidade ou empresarial. Os colaboradores incluem grupos e pessoas de contribuíram 
com a decisão. Mesmo uma decisão pessoal pode incorporar a participação do grupo, pois decisores individuais podem agir sozinhos ou basear sua decisão nos conselhos de outras pessoas (Franz \& Kramer, 2010). Dean e Sharfman (1996) argumentam que uma cultura na qual as ideias estratégicas são livremente defendidas e contestadas por qualquer pessoa pode ser um fator-chave na geração de estratégias organizacionais viáveis.

Além da quantidade e do papel das pessoas que participam de uma decisão, Cohen, March e Olsen (1972) identificaram variações em termos de tempo e energia empreendidos pelos participantes. Dessa forma, diferentes graus de envolvimento podem indicar diferentes graus de participação dos indivíduos na decisão. Decisões classificadas como de alto envolvimento são caracterizadas por alto nível de interesse, atividade e interação geradas pelo processo de decisão. De maneira complementar, Dholakia e Bagozzi (2002) indicam que indivíduos investem tipos diferentes de recursos no processo de decisão. Esses recursos podem incluir o investimento de tempo para pesquisar e coletar informações a respeito de alternativas disponíveis, o esforço cognitivo para memorizar e organizar as informações armazenadas na memória, o engajamento em debates para comparar e escolher entre alternativas e o esforço físico necessário à acumulação de informação relevante. O investimento combinado de todos esses esforços, incluindo o capital necessário à concretização das iniciativas, constitui o esforço investido pelo decisor durante o processo de decisão (Dholakia \& Bagozzi, 2002).

\subsection{EFETIVIDADE DA DECISÃO}

Quanto à efetividade da decisão - aqui entendida como a comparação entre os efeitos obtidos e os efeitos pretendidos pela decisão -, pode-se percorrer um continuum entre decisões com alto grau de efetividade e decisões com baixo grau de efetividade.

March e Simon (1958) avaliam como forma ideal de mensuração do sucesso de uma decisão, conforme proposta de teóricos economistas, a capacidade do decisor de maximizar seus efeitos em função de sua curva de utilidade. Contudo, em função de informações imperfeitas, capacidade 
cognitiva limitada e fatores não considerados, esse tipo de mensuração demonstra ser impossível no mundo real. Como alternativa, Keeney (2004) propõe como decisões efetivas aquelas que, em média, têm consequências positivas, admitindo que até mesmo decisões efetivas podem não garantir consequências positivas por causa das incertezas envolvidas. Dean e Sharfman (1996, p. 372) definem a eficácia de uma decisão estratégica como "o grau em que uma decisão atinge os objetivos estabelecidos pela administração no momento em que é executada". Contudo, a literatura sugere ser pouco preciso associar o resultado das decisões aos resultados organizacionais (Elbanna, 2006). Nutt (2001) argumenta que mais de 40\% das decisões organizacionais deixam de ser implementadas e muitas decisões implementadas têm valor questionável. Ou seja, um resultado negativo não pressupõe necessariamente uma decisão ruim, na medida em que mesmo uma decisão eficaz pode gerar resultados negativos em virtude de problemas em sua implementação.

\section{DISCUSSÃO DOS RESULTADOS}

As subseções a seguir apresentam discussões relacionadas a aplicações do esquema de classificação multidimensional do constructo decisão estratégica sob o ponto de vista (i) das formas de conceituação e de operacionalização do fenômeno, e (ii) da construção de tipologias de decisão estratégica.

\subsection{APLICAÇÕES DO ESQUEMA DE CLASSIFICAÇÃO MULTIDIMENSIONAL NA CONCEITUAÇÃO E OPERACIONALIZAÇÃO DO CONSTRUCTO DECISÃO ESTRATÉGICA}

As dimensões propostas e descritas na seção anterior permitem uma ampliação na compreensão do constructo decisão estratégica na medida em que descrevem o fenômeno de forma sintética e abrangente. Contribuem, portanto, para o desenvolvimento de críticas consistentes em relação às formas tradicionais de conceituação e operacionalização do constructo em trabalhos da área. Demonstram, ainda, a insuficiência de conceitos desenvolvidos com base em visões unidimensionais do fenômeno. Não seria 
suficiente, portanto, considerar como estratégica uma decisão tomada por indivíduos localizados no topo da hierarquia organizacional, sem que se considere também a importância ou abrangência da decisão. Também não seria suficiente considerar como estratégica uma decisão tomando-se como base sua efetividade, sem considerar a abrangência ou importância dos objetivos motivadores da tomada de decisão. Há, portanto, um campo fértil de aplicações do esquema de classificação proposto no sentido de se identificarem as relações de interdependência entre as dimensões e as formas de aplicação das múltiplas combinações possíveis na construção de conceitos teórica e empiricamente consistentes.

O esquema proposto também permite observar a importância da integração entre as perspectivas de conteúdo e de processo estratégico na medida em que integra aspectos relacionados às duas formas de abordagem. Por exemplo, ao propor-se a dimensão participação na decisão como elemento constitutivo da decisão estratégica, inclui-se uma preocupação com a quantidade e diversidade de interesses, percepções, papéis, graus de envolvimento ou opiniões consideradas na tomada de decisão. Considerando-se que esta dimensão pressupõe um continuum entre decisões com alto grau de participação e decisões com baixo grau de participação, evita-se a armadilha operacional da dicotomia indivíduoorganização e abre-se espaço para a construção de propostas de conceituação e operacionalização alinhadas com diferentes contextos empíricos, abordagens epistemológicas e bases teóricas.

Adicionalmente, as definições propostas na descrição das dimensões do esquema de classificação multidimensional permitem vislumbrar possibilidades de desenho de pesquisas com base nos elementos considerados. Por exemplo, em que medida a percepção de dificuldade da decisão estaria associada ao grau de participação em termos de quantidade de opiniões consideradas na tomada de decisão? Ou, ainda, em que medida o grau de efetividade da decisão estaria associado ao grau de estrutura da tomada de decisão? Essas e outras questões poderiam dar origem a investigações de natureza teórico-empírica potencialmente explicativas do fenômeno decisão estratégica sob múltiplas perspectivas, conforme apontado anteriormente. 


\subsection{APLICAÇÕES DO ESQUEMA DE CLASSIFICAÇÃO MULTIDIMENSIONAL NO DESENVOLVIMENTO DE TIPOLOGIAS DE DECISÃO ESTRATÉGICA}

As dimensões propostas do esquema de classificação multidimensional contribuem para ampliação da compreensão do fenômeno decisão estratégica ao permitirem a seleção e a combinação de atributos aplicáveis ao desenvolvimento de tipologias de decisão estratégica.

Por exemplo, tipologias com base no grau de estruturação da decisão poderiam contribuir para a identificação de tipos de decisões estratégicas relacionadas ao grau de intencionalidade na escolha do momento de tomada de decisão. Se há um momento ideal para tomada da decisão estratégica, conforme sugerem Harrison e Pelletier (1998), as decisões poderiam ser classificadas com base na possibilidade de escolha intencional - por parte do decisor - do momento de tomada de decisão associado à maximização dos efeitos pretendidos. Também poderia ser empregado o critério de grau de estruturação da decisão no desenvolvimento de tipologias de decisão com base no grau de racionalização do processo de tomada de decisão. Por exemplo, decisões de natureza híbrida (racionalintuitivas) poderiam ser classificadas com base no grau de racionalidade empregado na estrutura da tomada de decisão.

\section{CONSIDERAÇÕES FINAIS}

Diversos estudos têm falhado em captar a natureza multidimensional em face da complexidade e variedade do fenômeno decisão estratégica (Elbanna, 2006; Papadakis et al., 2010). Espera-se que as discussões apresentadas neste trabalho contribuam para o aperfeiçoamento das formas de conceituação e operacionalização do constructo. Não se trata da defesa por convergência ou unidade entre as pesquisas com foco em processo decisório, mas da defesa por coerência entre as bases epistemológicas e teóricas utilizadas, considerando-se a complexidade do fenômeno e a tendência a simplificações, conforme identificadas em estudos anteriores (Langley et al., 1995; Elbanna, 2006; Nutt \& Wilson, 2010, Nutt, 2011; Budde et al., 2015). 
Espera-se, ainda, estimular estudos futuros voltados, por exemplo, (i) à discussão de outras formas de classificação multidimensional do constructo decisão estratégica; (ii) ao desenvolvimento de subdimensões e indicadores aplicáveis às categorias propostas e (iii) ao desenvolvimento de estudos teórico-empíricos voltados à aplicação e revisão das dimensões propostas.

\section{REFERÊNCIAS}

Bandura, A. (1995). Exercise of personal and collective efficacy in changing societies. In A. Bandura (1995), Self-efficacy in changing societies (pp. 1-45). New York: Cambridge University Press.

Bardin, L. (2011). Análise de conteúdo. Lisboa: Edições 70.

Barnard, C. I. (1968). The functions of the executive (30th Anniversary Edition). Cambridge: Harvard University Press.

Bailey, K. D. (1994). Typologies and taxonomies: an introduction to classification techniques. Sage University Paper series on Quantitative Applications in the Social Sciences. Thousand Oaks, California: Sage.

Budde, L., Nagler, O., \& Friedli, T. (2015). A method to set up a complexity index to improve decision-making performance. Procedia CIRP, 36, 5358.

BUTLER, Judith. "The Force of Fantasy: Mapplethorpe, Feminism, and Discursives Excess". Differences: A Journal of Feminist Cultural Studies, vol. 2 (2), pp. 105-125, 1990.Chia, R. (1994). The concept of decision: a deconstructive analysis. Journal of Management Studies, 31(6), 781-806.

Child, J., Elbanna, S., \& Rodrigues, S. (2010). The political aspects of strategic decision making. In P. C. Nutt, \& D. C. Wilson (Eds.), Handbook of decision making (pp. 105-137). New Jersey: John Wiley \& Sons.

Clemen, R. T. (1996). Making hard decisions. Belmont, CA: Duxbury Press.

Cohen, M. D., March, J. G., \& Olsen, J. P. (1972, March). A garbage can model of organizational choice. Administrative Science Quarterly, 17(1), $1-25$.

Dean, J. W., \& Sharfman, M. P. (1996). Does decision process matter? A study of strategic decision-making effectiveness. Academy of Management Journal, 39(2), 368-392.

Dean, J. W., Sharfman, M. P., \& Ford, C A. (1991). Strategic decisionmaking: a multiple context framework. In J. Meindl, R. Cardy, \& W. 
Puffer (Eds.), Advances in Information Processing in Organizations (vol. 4, pp. 77-110). Greenwich: Jai Press.

Dholakia, U. M., \& Bagozzi, R. P. (2002). Mustering motivation to enact decisions. Journal of Behavioral Decision Making, 15, 167-188.

Eisenhardt, K. M. (1989). Making fast strategic decisions in high-velocity environments. Academy of Management Journal, 32(3), 543-576.

Eisenhardt, K. M. (1999). Strategy as strategic decision-making. Sloan Management Review, 40(3), 65-72.

Eisenhardt, K. M., \& Zbaracki, M. J. (1992, Winter). Strategic decision making. Strategic Management Journal, 13(Special Issue), 17-37.

Elbanna, Said. (2006). Strategic decision-making: process perspectives. International Journal of Management Reviews, 8(1), 1-20.

Franz, L. S., \& Kramer, M. W. (2010). The dimensions of decisions: a conceptual and empirical investigation. In P. C. Nutt, \& D. C. Wilson (Eds.), Handbook of decision making (pp. 154-196). New Jersey: John Wiley \& Sons.

Hambrick, D., Mason, P. (1984). Upper echelons: the organization as a reflection of its top managers. Academy of Management Review, 9(2), 193-206.

Hambrick, D. C., Finkelstein, S., \& Cannella Jr., A. A. (2009). Strategic leadership: theory and research on executives, top management teams, and boards. Oxford: Oxford University Press.

Harrison, E. F. (1996). A process perspective on strategic decision making. Management decision, 34(1), 46-53.

Harrison, E. F., \& Pelletier, M. A. (1998). Foundations of strategic decision effectiveness. Management Decision, 36(3), 147-159.

Hickson, D. J., Butler, R. J., Cray, D., Mallory, G. R., \& Wilson, D. C. (1986). Top decisions: strategic decision-making in organizations. Oxford: Basil Blackwell.

Keeney, R. L. (2004). Making better decision makers. Decision Analysis, $1(4), 193-204$.

Hodgkinson, G. P., \& Clarke, I. (2007). Conceptual note: exploring the cognitive significance of organizational strategizing: a dual-process framework and research agenda. Human Relations, 60(1), 243-255.

Langley, A., Mintzberg, H., Pitcher, P., Posada, E., \& Saint-Macary, J. (1995, May-June). Opening up decision making: the view from the black stool. Organization Science, 6(3), 260-279.

March, J. G., \& Simon, H. A. (1958). Organizations. New York: Wiley. 
Mintzberg, H., Raisinghani, D., \& Theoret, A. (1976, June). The structure of 'unstructured' decision processes. Administrative Science Quarterly, 21(2), 246-275.

Mintzberg, H., Otis, S., Shamsie, J., \& Waters, J. A. (1988). Strategy of design: a study of architects in co-partnership. In J. Grant (Ed.), Strategy Management Frontiers (pp. 311-359). Greenwich, CT: JAI Press.

Mintzberg, H., \& Waters, J. (1990). Studying deciding: an exchange of views between Mintzberg and Waters, Pettigrew, and Butler. Organization Studies, 11(1), 1-16.

Moody, P. E. (1983). Decision making: Proven methods for better decisions. New York: McGraw-Hill Book.

Nutt, P. C. (1998). Evaluating complex strategic choices. Management Science, 44(8), 1148-1166.

Nutt, P. C. (2001). A taxonomy of strategic decisions and tactics for uncovering alternatives. European Journal of Operational Research, $132(3), 505-527$.

Nutt, P. C. (2011). Making decision-making research matter: some issues and remedies. Management Research Review, 34(1), 5-16.

Nutt, P. C., \& Wilson, D. C. (2010). Crucial trends and issues in strategic decision making. In P. C. Nutt, \& D. C. Wilson (Eds.), Handbook of decision making (pp. 3-29). New Jersey: John Wiley \& Sons.

Papadakis, V., \& Barwise, P. (1998). Strategic decisions: an introduction. In: Papadakis, V., \& Barwise, P. (eds). Strategic decisions: context, process and outcomes (pp. 1-16). London: Kluwer Academic Press.

Papadakis, V., Thanos, I., \& Barwise, P. (2010). Research on strategic decisions: taking stock and looking ahead. In P. C. Nutt, \& D. C. Wilson (Eds.), Handbook of decision making (pp. 31-70). New Jersey: John Wiley \& Sons.

Pennings, J. M. (1985). Introduction: on the nature and theory of strategic decisions. In J. M. Pennings, Organizational strategy and change ( $\mathrm{pp}$. 134). San Francisco: Jossey-Bass.

Pettigrew, A. M. (1990). Studying strategic choice and strategic change: a comment on Mintzberg and Waters: 'does decision get in the way?'. Organization Studies, 11(2), 6-11.

Pettigrew, A. M. (1992). The character and significance of strategy process research. Strategic Management Journal, 13(S2), 5-16.

Pfeffer, J. (1981). Power in organizations. Marshfield, MA: Pitman.

SHEPHERD, N.G.; RUDD, J.M. The influence of context on the Strategic decisionmaking process: a review of the literature. International Journal 
of Management Reviews, v. 16, p. 340-364, 2014.Shepherd \& Rudd (2014)SIMON, H.A. Making management decisions: The role of intuition and emotion. Academy of Management Executive, February, p.57-64, 1987.

SIMON, H.A. et al. Decision making and problem solving. Management science. v.17, n.5, p.11-21, 1987.

Tsoukas, H. (2010). Strategic decision making and knowledge: A Heideggerian approach. In P. C. Nutt, \& D. C. Wilson (Eds.), Handbook of decision making (pp. 379-402). New Jersey: John Wiley \& Sons.

Wilson, D. (2003). Strategy as decision making. In S. Cummings, \& D. Wilson (Eds.), Images of strategy (pp. 383-410). Oxford: Blackwell.

Yates, J. F. (1990). Judgment and decision making. New Jersey: PrenticeHall. 